\title{
LA SUCESIÓN ENTRE LOS INCAS
}

\author{
SUCCESSION AMONG THE INCAS
}

\author{
Francisco Hernández Astete ${ }^{1}$
}

\begin{abstract}
Uno de los temas más controversiales en lo que se refiere a la organización andina prehispánica es el de la sucesión en el mando. Los incas no son una excepción en este sentido y desde el siglo XVI se ha planteado la interrogante y se ha esbozado más de un teoría. El tema no ha sido ajeno a los cronistas de Indias ni a los historiadores modernos.

Básicamente se han planteado dos posiciones. De un lado, está aquella que entiende la información de las crónicas andinas textualmente y presenta una visión de la sucesión incaica que incorpora a los Andes los lineamientos de la sucesión dinástica europea de la época. En esta postura intervienen -entre otros- los conceptos de bastardía, legitimidad y primogenitura. De otro lado, se ha desarrollado otro planteamiento que pretende ser más crítico con las fuentes y plantea una estructura alternativo. Así, desde la Etnohistoria, la tesis de la "habilidad" para gobernar ha cobrando fuerza como uno de los lineamientos principales en el tema de la sucesión incaica. En el presente artículo se revisan los argumentos planteados hasta el momento sobre el tema de la sucesión, y se ofrece, a la luz de las fuentes coloniales sobre los Andes, una interpretación alternativa en la que la sucesión del poder a los hermanos del gobernante parece ser previa al paso del mismo a la siguiente generación
\end{abstract}

Palabras claves: sucesión, incas, Andes, Tahuantinsuyo, poder, Etnohistoria, crónicas.

One of the most controversial subjects with regard to the Andean pre Hispanic Organization is the one referring to succession of command. The Incas are not an exception on this matter, and since the sixteenth century the query has been raised and more than one theory has been devised. The subject has not been alien to chroniclers of the Indias nor to modern historians.

Basically, two postures have arisen. On the one hand, there is one that interprets the information of the Andean chronicles literally and presents a vision of Inca's succession that incorporates to the Andes, the parameters of the European dynastic succession of the epoch. In this posture intervenes, among others, the concept of bastardy, legitimacy and primogeniture.

On the other hand, another posture has been developed, aiming to be more critic with the sources and to offer an alternative structure. From the Ethnohistoric point of view, the thesis on the "ability" to govern has become stronger as a principal parameters on the subject of Inca's succession. This article reviews the postures proposed on the subject of succession and based on the colonial sources on the Andes. I present an alternative interpretation in which the power from the King to his brothers seems to be previous to the stage of passing power to the next generation.

Key words: Succession, Incas, Andes, Tahuantinsuyo, power, Ethnohistory, chronicles.

Las primeras versiones que se escribieron acerca de la sucesión entre los incas privilegian una postura dinástica sobre el tema en la medida en que "traducen" la información que los cronistas dan a la hora de escribir las biografías de los incas. De esta forma, se afirma que se heredaba el "señorío" de padres a hijos, respetando el principio de primogenitura y planteando, además, el asunto de la habilidad para gobernar como la excepción que confirmaría la regla para casos en los que el legítimo heredero tuviera serios problemas físicos para acceder de manera plena al ejercicio cotidiano del poder. Es, sin embargo, desde mediados del siglo pasado que la historiografía sobre los incas se fue alejando de aquella posición. Así, María Rostworowski, en 1956, con su trabajo sobre Pachacútec -reelaborado posteriormente en 1983- afirma que serían los hijos de cualquier inca, y no necesariamente del gobernante anterior; es decir, los miembros de las llamadas "panacas", los que podrían acceder a la borla dependiendo de su capacidad para gobernar (Rostworowski 2001[1953],1983). Adicionalmente, Rostworowski plantea como uno de los mecanismos sucesorios el tema del correinado entre los incas;

1 Pontificia Universidad Católica del Perú, Lima, Perú. fhernan@ @ucp.edu.pe 
el mismo que entiende como el gobierno conjunto del inca y su heredero principal en una suerte de entrenamiento para el mando. De esta forma, los incas habrían encontrado la mejor solución para un viejo problema. Así, para esta autora, en su primer planteamiento, la elección del gobernante se producía durante el gobierno del padre: "la regla, que parece haber existido en el momento culminante del incario, fue la elección del más hábil entre los candidatos al gobierno, debiendo tener preferencia los vástagos de la coya, por ser la mujer principal" (Rostworowski 2001 [1953]:240). Es necesario resaltar también que, para esta autora, el sistema del correinado no implicó una obligación vinculante con la persona elegida para la sucesión en el mando, por lo que la decisión podría ser revocada en cualquier momento de acuerdo a las habilidades que demostrara durante esta suerte de periodo de prueba (Rostworowski 2001 [1953]:240). Esta postura fue, como se ha mencionado, ampliada posteriormente por la misma autora-concretamente en 1983- en un trabajo destinado al estudio de las estructuras políticas y religiosas incaicas. En este estudio, Rostworowski amplió las evidencias que confirmaban sus hipótesis iniciales acerca del vínculo entre la sucesión y el correinado, afirmó la tesis de la "habilidad" en el mando como requisito fundamental para acceder al poder y amplió a todos los miembros de la élite -los integrantes de las llamadas panacas- la posibilidad de convertirse en incas. Es necesario resaltar además que, en este trabajo, señaló, como detallaremos más adelante, la importancia del aillu materno como uno de los criterios más importantes a la hora de la elección del soberano del Cuzco (Rostworowski 1983:167 y ss).

Por su parte, aunque nunca abordó el tema de manera monográfica, Franklin Pease G.Y. mencionó siempre la necesidad de entender la sucesión entre los incas desde una perspectiva distinta a la de la norma europea vigente hacia 1500. En este sentido, motivado por la asociación entre correinado y sucesión presentada por María Rostworowski, analizó la información que sobre el tema presenta Sarmiento de Gamboa. Verificó con ella que en todas las sucesiones narradas por el cronista existieron siempre dos candidatos, uno de Hanan y otro de Rurin $^{1}$, asociándose el primero con las conquistas fuera del Cuzco, y el segundo con la administración de la ciudad sagrada. Asimismo, notó cómo es una constante en la obra de Sarmiento el hecho de que el candidato designado por el inca nunca asumía el mando, lo que consideró una prueba adicional de la necesidad de combinar el "derecho" a gobernar con la habilidad para ejercer el mando (Pease 1991:96-97). Adicionalmente, muchos otros autores se han referido al tema de la sucesión desde esta perspectiva, manteniendo básicamente la postura de una designación basada en la habilidad en el mando sin desarrollar una posición alternativa ${ }^{2}$. Quizás, la postura más distanciada de esta tendencia, asociada básicamente con la sucesión de Huaina Cápac, y sobre la que volveremos más adelante, es la de Concepción Bravo (1976).

Así, existe solo un trabajo destinado íntegramente a responder la pregunta acerca de la sucesión entre los incas, el realizado por Liliana Regalado de Hurtado (1996 [1993]). En ese trabajo, basado en la información de Juan de Betanzos, la autora escribe una importante monografía sobre el tema destacando dos cuestiones primordiales. Por un lado, la habilidad de las madres para hacer que uno de sus hijos se convierta en el inca. Por otro, la necesaria aprobación divina que vendría apoyada por la ceremonia de la callpa, y de una serie de procesos rituales que tendrían la misión de elegir al candidato de peso para hacerse con la borla como un mecanismo de manifestación de la posición de la élite acerca de los distintos candidatos.

En sus planteamientos, Regalado de Hurtado (1996 [1993]) asume básicamente los postulados anteriormente desarrollados sobre el tema por Tom Zuidema y, sobre todo, por María Rostworowski, aunque elabora una propuesta más amplia a partir de la información de Betanzos. En esta propuesta incluye, básicamente, un complejo proceso sucesorio en el que se seguirían las siguientes etapas: manifestación de señales divinas a favor de uno de los candidatos, la capacidad del futuro inca para obtener el control de los recursos del área, el incremento de la élite a partir de la celebración de convenientes matrimonios, la asociación con un grupo importante de la élite y el dominio territorial expresado en las conquistas realizadas por el aspirante a la borla colorada. Asimismo, menciona Regalado de Hurtado (1996 [1993]) que, además de las actividades rituales que el futuro gobernante debía realizar -entre las cuales destacan la celebración de las exequias de su padre, así como la realización de un baño ritual antes de asumir el mando-, se asociaban con el proceso sucesorio y con la definición de los candidatos las prácticas de diversas actividades destinadas a hacerse con el control definitivo del poder. Estas estaban, 
principalmente, asociadas con el reordenamiento del Cuzco, el control de diversas poblaciones, la realización de conquistas exitosas y la ocupación ritual y "reconquista" del Cuzco que finalizaba el proceso. Como es evidente, esta autora, como veremos más adelante, incorpora también en su trabajo la idea de la dualidad en el mando y la del correinado como mecanismo sucesorio (Regalado de Hurtado1996 [1993]).

Aun cuando muchos de los trabajos que analizan el tema de la sucesión suscriben también la tesis de la dualidad en el mando, sus conclusiones acerca de la transmisión del mismo parecen corresponder a un solo proceso en el que se elige a un único gobernante, dejando de lado la posibilidad de que se eligiera a dos candidatos de manera separada. Como se ha mencionado, Rostworowski asume el correinado como un mecanismo sucesorio, aunque en sus textos también aparece la idea de un correinante contemporáneo del supuesto inca principal. Por su parte, dentro de la posición de Regalado de Hurtado (1996 [1993]), la condición de Hanan o Rurin para los incas sería únicamente el resultado final de una única lucha por el poder. En las páginas que siguen intentaremos presentar algunas ideas acerca de la sucesión incaica.

\section{La Influencia de la Élite en la Elección del Nuevo Inca}

Como se ha adelantado ya, es a través del estudio de la influencia de las mujeres en el proceso sucesorio que la historiografía sobre los incas ha mostrado la injerencia de la élite en el ejercicio del poder. Una primera aproximación a este problema fue planteada por María Rostworowski al estudiar el rol sociopolítico de las mujeres incas y encontrar que jugaban una función importante en las estructuras de las llamadas panacas, puesto que a través de la parentela materna los aspirantes a incas hicieron valer sus derechos a la sucesión (Rostworowski 1983:168). Posteriormente, la misma autora definió la influencia de la parentela femenina en la sucesión incaica mediante un análisis de la intervención de las madres de Huáscar y Atahualpa en el último proceso sucesorio del que se tiene noticia (Rostworowski 1988:137-157). Como se mencionó, el tema fue ampliado por Liliana Regalado de Hurtado (1996 [1993]), quien afirma que, sin constituir un criterio absoluto y aunque bastaba tener por madre a una de las integrantes de la élite incaica para convertirse en inca, los hijos de la coya, si además eran hábiles y capaces, tenían mayor posibilidad de acceder al poder pese a la gran cantidad de hijos del inca (Regalado de Hurtado1996 [1993]:48). Adicionalmente, en un trabajo anterior, señalamos que un criterio importante en la definición de la sucesión estaba relacionado con la mujer que el candidato podría presentar como coya. Es decir, no solo era importante tener una madre influyente, sino también haber conseguido una esposa capaz de aportar una valiosa alianza al candidato (Hernández Astete 1997, 2002a).

Evidentemente, la importancia de la mujer, hermana, madre o esposa, dentro del proceso sucesorio, se da a partir de la parentela que hay en torno a ella, pues los distintos linajes que integraban la nobleza cuzqueña continuaban accediendo a los privilegios obtenidos por medio del parentesco de su ancestro fundador, incluso en pleno proceso sucesorio. De ese modo, madre y esposa significaban para el candidato a inca el apoyo de los linajes a los que aquellas mujeres pertenecían: el mismo que se materializaba en el control de población y, por lo tanto, en la capacidad para convocar las mitas que permitieran al futuro inca consolidarse como gobernante del Cuzco.

Es por esta razón que el futuro inca buscaba aliados a través de la celebración de matrimonios al interior de la élite cuzqueña. Es en este sentido ilustrativo el caso que presenta Pachacuti al mencionar que Huaina Cápac:

pretende casarse con su segunda ermana carnal llamada Mama Coca, el qual su ermana no consiente el cassamiento, y por su ermano visto aquello, les maltrata y pretende hazer fuerça, y no los puede assí por ruegos y amenazas. Entonces va con ofrendas al cuerpo de su padre a pedir que les "las diese por mujer" y el muerto nunca responde [énfasis mío] (Pachacuti 1993:246).

El hecho de que el muerto no respondiera, decisión que debió comunicar la pareja encargada de interpretar las decisiones del ancestro, expresa claramente la negativa del linaje ante la alianza propuesta $^{3}$. El caso es sumamente sugerente, pues muestra que era posible que un aillu cuzqueño rechazara el ofrecimiento de matrimonio por parte del futuro inca, posiblemente porque el grupo estaba interesado en una alianza con un bando distinto. 
La búsqueda de aillus reales aliados en el proceso sucesorio a través de matrimonios ${ }^{4}$ es ilustrada, también, por Juan de Betanzos cuando menciona que Huaina Cápac adopta por madre a una hija de Yamque Yupanqui, visiblemente su segunda persona según el citado cronista, para entregarla luego por mujer a su hijo Atahualpa (Betanzos1987 [1571]:197) ${ }^{5}$. Cabe anotar que esta situación que merece un análisis mayor desde el punto de vista del parentesco incaico, pues que una mujer sea, simultáneamente, abuela y esposa, evidencia la alianza entre abuelos y nietos, bastante documentada en la mitología andina contemporánea ${ }^{6}$. Por otra parte, el mismo cronista, al hablar de Pachacútec, muestra cómo era importante para el mantenimiento del poder que el inca tomara esposas secundarias tanto de Rurin como de Hanan Cuzco:

\section{"[...] y ansi mismo en este tiempo tomó por mujeres otras veinte señoras hijas de aquellos principales de la ciudad ansi de los Huren Cuzco como los de Hanan Cuzco [...]" (Betanzos1987 [1571]:99-100).}

Ello, como es evidente, sugiere una alianza con todos los aillus cuzqueños -según la versión de Juan de Betanzos, diez de Rurin y diez de Hanan-.

Mencionamos en un trabajo anterior (Hernández Astete 2010) la posibilidad de que la figura del "capitán" señalada por Guaman Poma de Ayala ${ }^{7}$ sugería la existencia de una suerte de periodo de prueba para los probables incas; información que concuerda con la hipótesis del correinado planteada inicialmente por Rostworowski y ampliada posteriormente por Liliana Regalado de Hurtado (1996 [1993]). En este sentido, la idea de que la figura del capitán funcione dentro del proceso sucesorio se percibe también en el hecho de que casi todos los capitanes que el cronista menciona están incorporados tanto en la lista de hijos de las coyas, como en la que se refiere a los hijos de los incas. Asimismo, todos los capitanes a los que se refiere Guaman Poma de Ayala, hasta antes de Huaina Cápac, murieron en vida del inca reinante, en medio de aparentes procesos de expansión. Ese es, por ejemplo, el caso de "Inga Mayta" e "Inga Urcón", hijos de Yahuar Huaca en la versión del cronista andino, sobre los que manifiesta que "[...] murieron todos después de la conquista en la Ciudad del Cuzco, por donde le pesó muy mucho a su padre y fue enterrado muy honradamente" (Guaman Poma 1993 [1613]:122). En este caso, nos encontramos ante un ciclo sucesorio no completado, dado que aparentemente no habrían terminado la conquista, pues mueren en el Cuzco. Vale la pena recordar que, en varios de sus trabajos, Pease menciona que luego de asumir la borla, el inca efectuaba una conquista ritual que terminaba con una exitosa reconquista del Cuzco. En este caso, se podría pensar que tal conquista tenía lugar también antes de asumir el poder y como parte del proceso de sucesión. Atahualpa, por ejemplo, se encontraba fuera del Cuzco cuando aún no había concluido la sucesión. Otros casos en los que no se completa la sucesión por parte de los capitanes mencionados por Guaman Poma, son los de Inga Urcón y Auqui Topa Inga, hijos de Topa Ynga Yupanqui. En este ciclo sucesorio, luego de mencionar que Inga Urcón fracasó en el intento de llevar una piedra del Cuzco a Huánuco $^{8}$, y que Auqui Topa Inga conquistó Huánuco, Caxatambo, Conchucos, Chinchaycocha, Tarma, Canta, Huno, Lurin y Hanan Guaylas, Guaman Poma dice que "[...] murió el primer capitán en el Cuzco, el segundo capitán murió en la conquista, murieron como valerosos capitanes [...]" (Guaman Poma 1993 [1613]:125). Estos capitanes estarían disputándose la borla, pero habrían fracasado en el proceso, razón por la cual no llegaron a ser incas. Además, resulta importante mencionar-pensando en futuras investigaciones al respecto -que la relación de los mismos sugiere que siempre eran dos los que estaban en guerra por la borla, noción que concuerda con la guerra ritual en la que Huáscar y Atahualpa estuvieron envueltos luego de la muerte de Huaina Cápac.

De esta manera funcionaría entonces como parte del proceso sucesorio, una suerte de mecanismo de prueba en el que los probables incas -cuyos derechos estaban asociados con su pertenencia al linaje realpodrían probar sus habilidades para ejercer el cargo, dado que resulta bastante claro que no bastaba ser el "primogénito" ni poseer la condición de noble para gobernar el Tahuantinsuyo, sino que existió un proceso de designación, asociado con la búsqueda de los candidatos con mayores capacidades dentro de los nobles cuzqueños.

Un ejemplo de esta situación es también el que nos ofrece Garcilaso de la Vega al narrar el gobierno de Inca Roca y la forma en que encargó a Yahuar Huaca la conquista del Antisuyo:

Pasados algunos años que el Rey Inca Roca gastó en paz y quietud en el gobierno de 
sus reinos, le pareció enviar al príncipe heredero llamado Yahuar Huacac su hijo, a la conquista de Antisuyo, que es levante del Cozco y cerca de la ciudad; porque por aquella banda no se había alargado su imperio más de lo que el primer Inca Manco Cápac dejó ganado hasta el río Paucartampu (Garcilaso 1960[1609]:136).

Asimismo, resulta ilustrativo el ciclo sucesorio de Pachacútec que ofrece en su crónica el Inca Garcilaso de la Vega. En él, refiere el cronista que es Cápac Yupanqui el responsable de muchas de las conquistas en la época de Pachacútec, evidentemente su segunda persona, las mismas que se organizan en ciclos de tres años. En este contexto, afirma que, tras una visita general que dura tres años,

se volvió a su corte, donde gastó algunos meses en fiestas y negocios; mas luego trató con el hermano, que era su segunda persona y con los de su consejo de volver a la conquista de las provincias de Chinchaisuyo [...] Acordaron que el Inca Capac Yupanqui volviese a la conquista, pues en la jornada pasada había dado tan buena muestra de su prudencia y valor, y de las demás partes de gran capitán; mandaron que llevase consigo al príncipe heredero su sobrino, llamado Inca Yupanqui, muchacho de diez años [...] [énfasis mío] (Garcilaso1960 [1609]:209).

A su vez, y en oposición con la idea de un inca todopoderoso, Liliana Regalado de Hurtado ha planteado la hipótesis de la existencia de una doble diarquía en el gobierno incaico. La primera diarquía, integrada por los ya conocidos incas Hanan y Rurin; y la segunda, asociada a miembros de una generación posterior a la primera y con menor rango que los anteriores (Regalado de Hurtado1996 [1993]). En ese sentido, se considera que la segunda diarquía no estaría totalmente consolidada y se asociaría más a esta suerte de "sistema de entrenamiento", donde los futuros incas demostraban sus habilidades para hacerse con el poder (Hernández Astete 2002b). Ese sería, por ejemplo, el caso de Yamque Yupanqui y el de Túpac Inca, quienes -según Betanzos- estaban encargados de realizar conquistas aún en vida de Pachacútec (1987 [1571]:127). Ambos, cabe anotar, fueron posteriormente convertidos en incas, experiencia distinta de aquellos que fallaron en sus expediciones de conquista, como Amaro Túpac Inca y Paucar Ushno, quienes fracasaron en la expedición a Collasuyo que terminó con la muerte del segundo (Betanzos 1987 [1571]:120)9.

De hecho, la sucesión de Pachacútec en la obra de Betanzos merece un análisis mayor, pues según el cronista, a su muerte, no solo quedaron Yamque Yupanqui y Túpac Inca Yupanqui en el poder, sino que ya había nacido Huaina Cápac y lo habían convertido en inca, aunque en una evidente posición inferior con respecto a los anteriormente mencionados (Betanzos 1987 [1557]:127-129).

Así, aunque Betanzos afirma que a la muerte de Pachacútec, Túpac Inca Yupanqui y Huaina Cápac quedaron en el poder, se desprende del propio texto la autoridad de Yamque Yupanqui pues además de estar, en opinión del cronista, a cargo de la educación de Huaina Cápac, Pachacútec le encarga también “...el gobierno de todo el reino mientras viviese pues su hijo Topa Ynga siempre había de andar en las guerras..." (Betanzos 1987:137).

Resulta evidente que la referencia anterior no corresponde a la asignación de un tutor o regente para Huaina Cápac -idea que es a todas luces un sesgo occidental de la información-, sino que da cuenta de parte del proceso sucesorio en el que Yamque Yupanqui quedaba convertido en una suerte de ancestro ejemplar, en posición Rurin dentro del esquema de la doble diarquía de acuerdo a lo que planteó anteriormente Regalado de Hurtado (1996 [1993]).

De este modo, podría pensarse, en concordancia con Concepción Bravo (1992:47), que Túpac Yupanqui sería el inca Hanan; y Yamque Yupanqui, el Rurin. Por eso, se vincula al primero principalmente con las guerras. Sin embargo, las funciones de Yamque Yupanqui no son estrictamente sacerdotales, sino que se encuentran asociadas directamente con la administración. Probablemente, por esto existía, según los informantes de Betanzos, un acuerdo en la élite para después de la muerte de Yamque Yupanqui: "nombraban por gobernador y en lugar de su señor Topa YngaYupangue a un hermano suyo que se decía SoponoYupangue (Betanzos 1987 [1571]:155).

En la cita anterior da cuenta de que, aun cuando queda clara la necesidad de reemplazar al inca Rurin, aparentemente también sería reemplazado Túpac Inca, por lo que se podría pensar que Túpac Inca pasaba a asumir las funciones de Yamque 
Yupanqui. Adicionalmente, la sucesión del inca Rurin -tema que merece una mayor atención de los investigadores-, aparentemente, se relaciona con la referencia anteriormente mencionada sobre el hecho de que Huaina Cápac había adoptado como madre a la recién nacida, hija de Yamque Yupanqui, su segunda persona, e hijo del anterior correinante de Túpac Inca Yupanqui (Pachacútec). En este sentido, si aceptamos la existencia de la cuatripartición en el poder incaico, que entiende la existencia simultánea de "cuatro incas" -dos en la primera diarquía y dos en la segunda-, habría que preguntarse si esta implicaba o no la presencia de cuatro linajes en el poder, y si estos cuatro incas estarían asociados con la división en cuatro suyos tan documentada, pero no necesariamente cierta, para el país de los incas. Sobre el tema, como se ha resaltado, Guaman Poma de Ayala -luego de escribir sobre incas y coyas- menciona la existencia de capitanes y señoras, e identifica a los capitanes como las segundas personas del inca, y a las señoras como esposas de los últimos. Ese es, por ejemplo, el caso del tiempo de Huaina Cápac en la Nueva Corónica, pues además del inca y la coya, en este caso Raua Ocllo, el autor menciona a CápacApoGuamanChaua, capitán que identifica como segunda persona del inca, casado con una de las que denomina "señoras", CápacUarmi Poma Gualca. De este modo, según Guaman Poma, tendríamos esta estructura como la siguiente ${ }^{10}$ :

\begin{tabular}{ll}
\hline $\begin{array}{l}\text { Inca Hanan: } \\
\text { Huaina Cápac }\end{array}$ & $\begin{array}{l}\text { Capitán: } \\
\text { CápacApoGuamanChaua }\end{array}$ \\
\hline $\begin{array}{l}\text { Coya Hanan: } \\
\text { Raua Ocllo }\end{array}$ & $\begin{array}{l}\text { Señora: } \\
\text { CápacUarmi Poma Gualca }\end{array}$ \\
\hline
\end{tabular}

Ese habría sido, también, el caso de la sucesión de Huiracocha, quien estaba absolutamente desprestigiado tras su abandono del Cuzco ante la amenaza de los chancas, y la posterior derrota de los mismos por parte de Inca Yupanqui Pachacútec. En ese contexto, aun cuando Huiracocha insiste en que Inca Urco debía convertirse en el gobernante son -según Sarmiento- sus propios hijos y capitanes, ApuMaita y Vicaquirao, quienes se oponen a la voluntad del inca, lo que facilita el acceso de Inca Yupanqui Pachacútec al poder (Sarmiento1988 [1572]:83-84) ${ }^{11}$. El propio Sarmiento menciona, también, otros casos de sucesiones que cambiaron de destinatario como una manera de mantener la alianza con ciertos sectores de la élite. Pueden ilustrar esta situación los casos de Lloque Yupanqui, convertido en inca tras el desplazamiento de Manco Sapaca (Sarmiento 1988 [1572]:80); el de Túpac Inca Yupanqui, convertido en gobernante tras el desplazamiento de Inca Amaru (Sarmiento 1988 [1572]:115); y el de Huaina Cápac, quien queda convertido en inca luego de que Huaman Achachi lograra evitar que la familia de Cápac Huari se haga con el poder (Sarmiento 1988 [1572]:138-139).

Por otra parte, la evidente y natural interdependencia del inca con el sector más privilegiado e influyente de la nobleza cuzqueña -probablemente el Hanan- queda evidenciada en la narración de Sarmiento de Gamboa, pese a que la misma está hecha siguiendo los moldes occidentales de nombramiento del sucesor. Así, dice el cronista que Pachacútec, en los últimos momentos de su vida, reunió a sus hijos y a los orejones del Cuzco y dirigiéndose a Túpac Inca dice: "A estos nuestros deudos te dejo por padres, para que te aconsejen. Mira por ellos y ellos te sirvan" (Sarmiento1988 [1572]:126). Claramente, los informantes de Sarmiento se refirieron a un momento clave en las transformaciones políticas de la élite. Siguiendo a Regalado de Hurtado (1996 [1993]:64 y ss.), estaríamos ante una transformación de las diarquías, pues, luego de haberse asegurado la condición Hanan de Túpac Inca frente a Amaru Inca (Sarmiento 1988 [1572]:115), había llegado el momento del enroque generacional. Debido a ello, el correinante de Pachacútec quedaría en la segunda diarquía, mientras que Túpac Inca y Amaru Inca pasarían a integrar la diarquía principal.

\section{Un Modelo Alternativo}

Señaladas las tendencias que existen en la bibliografía acerca de la sucesión incaica, así como la influencia de la élite cuzqueña en este proceso, interesa ahora pensar en este asunto entendiéndolo en función de la necesidad de reemplazar dos posiciones interdependientes. Así, aunque la historiografía haya avanzado largamente sobre el tema, vale la pena seguir las evidencias de una alternativa diferente; la misma que nos pueda, además, ofrecer otros alcances sobre el proceso sucesorio incaico. Por este camino, existen dos pistas importantes que nos pueden llevar a construir un sistema complementario. Por un lado, el hecho 
de que la mayor parte de la información sobre la habilidad para gobernar como requisito casi único en la sucesión provenga de los llamados textos toledanos y, por el otro, la modificación del texto de Agustín de Zárate sobre la sucesión incaica luego de la realización de las informaciones toledanas y de la publicación de la historia de Sarmiento de Gamboa. Y es que, probablemente, el texto de Zárate se modificó a propósito de la consolidación de la elaboración de la historia oficial de los incas; la misma que terminó por prohibir la investigación sobre la historia de los naturales andinos con miras a perpetuar ese punto de vista.

Respecto del primer tema, como se ha manifestado ya, la cantera principal de evidencias acerca de la habilidad como requisito para gobernar el país de los incas está constituida por los textos comúnmente denominados como toledanos. Así, por ejemplo, el conocido Anónimo de Yucay dice sobre la sucesión:

Y son estos caçiques tan malos y tan viçiosos comúnmente que todo lo lleuantrás sí. Y si éstos suçediesen "jure ereditario", era destruiçión de todo el gouierno, porque era sufrir cauesas malas y peruersas que destruyesen la República, y quitalle al rey el poder de haçer elecçión de los mejores y virtuosos, y a ellos la ocaçión de pretender estos estados por virtud y buena christiandad. Porque, en sauiendo ellos que estos cazicazgos no se auían de dar sino a los mejores, criarían a sus hijos virtuosa y christianamente para que su Magestad se los diese. y si el hijo menor era mejor, darlo aquel, y si todos biciosos a ninguno, sino al mejor siempre. Y esta libertad tiene su Magestad agora, y ésta la quitaua este Padre, haziendo éstos señores naturales (Anónimo de Yucay, 1979 [1570]:114-115).

En este caso, no queda ninguna duda de que señalar la habilidad para el mando como requisito indispensable -quizás único- para el ejercicio del poder era una práctica que cierto sector de la burocracia Habsburgo en América sugería al rey que la mención a esta fuera incorporada a la legislación sobre el tema. Lo que evidentemente no queda claro es si se trataba de una práctica andina recogida por los funcionarios virreinales o, por el contrario, su incorporación a la historia de los Andes constituía un argumento adicional en el proceso de justificación de la conquista. Es importante en este sentido estudiar lo que nos dicen sobre el tema los documentos toledanos conocidos como las Informaciones sobre los Incas; los mismos que fueron realizados sobre la base de los interrogatorios a los que se sometió a los más importantes nobles cuzqueños en la década de 1570. Interesa, en primer término, analizar lo que planteaban sobre el tema de la sucesión las preguntas formuladas por los funcionarios virreinales a sus informantes andinos, dado que, al parecer, se estaba construyendo una categoría -bastante conveniente por cierto- sobre las costumbres y tradiciones andinas:

Sy es verdad que a estos no los elegiansynoquellos como valientes se señalauan en las guerras y todos los seguian como a tales (Levillier 1940:47).

Sy es verdad quelyngaponia los curacas conforme a lo que le parescia y segun las partes que hallava en ellos para el govierno de manera que si avia muchos hijos lo dava al que le parescia que teya mas partes para gobernar (Levillier 1940:47).

Sy es verdad que quando no avia [entre] los descendientes del curaca muerto persona que satisficiese al ynga para nombrarle, en su lugar nombrava otro qual le parescia fuese su deudo o no lo fuese [...] (Levillier 1940:47-48).

Preguntas como las transcritas, qué duda cabe, estaban orientadas a justificar, mediante los testimonios que motivaran, no solamente el hecho de que no existió un sistema sucesorio entre los curacas andinos, sino, sobre todo, que las autoridades étnicas eran nombradas por el inca sin seguir ningún patrón de sucesión y que, por lo tanto, estaba absolutamente justificada la idea de que el monarca hispano, a través de sus funcionarios, nombrara a los curacas coloniales. Estos datos, interpretados convenientemente y extendidos al Tahuantinsuyo, ayudarían también a contradecir las tesis de la legitimidad de las autoridades andinas y a justificar la conquista. Quizás, por esta razón, los cronistas desarrollan estos argumentos en sus redacciones, aunque señalan también algunas contradicciones al respecto, aplicando para los incas las normas de sucesión de la Europa moderna. De hecho, 
las propias conclusiones que presentan sobre el tema las Informaciones sobre los Incas permiten seguir la argumentación que se está presentando:

\begin{abstract}
Pueuaseansimismo que quandoauiaalgun hijo de tal curaca o caçique o principal muerto que tuviese abilidad y entendimiento para gouernar el dicho ynga le proveia en el dicho cargo sin tener consideración a que fuesse el mayor o el menor sino a el que tuviese masabilidad y que siempre estuvo en costumbre de quitar y poner estos Curacas y Caçiques como le parecia y en quien quería sin tener rrespeto a dezendencias ni subzesiones ni a otra cossa y no auía ninguna contradicción en ello (Levillier1940:5).

[...] que tiniendo vuestra Magestad el verdadero señorío destosrreynos como lo tiene y no aviendo como no hay ligitimossubzesores de los tiranos yngas todas las minas y minerales y todos los bienes del Sol $\mathrm{y}$ ydolos y todos los tesoros de sepulturas y tierras y ganados questan dedicados para seruicio de los cuerpos de los yngas en que no haya poseedores particulares con buen titulopertenencen a Vuestra Magestad como a rrey y Señor como bienes vacos mostrencos questanproderelictos (Levillier 1940:12).
\end{abstract}

$\mathrm{Al}$ parecer, bien podría entenderse que quienes estuvieron detrás de estos textos quisieron dar la imagen de una organización absolutamente caótica para el tema de la sucesión en los Andes; y es, de ese modo, que se podría justificar la conquista bajo el supuesto de haber derrocado a jefes carentes de legitimidad. En este caso, si pensamos, por ejemplo, en la información de Pedro Sarmiento de Gamboa, podríamos entender mejor el asunto. En este sentido, como recordábamos líneas atrás, Franklin Pease G.Y. destacó cómo este cronista, aunque abogaba por una sucesión dinástica, deslizaba en su obra datos importantes para corroborar la hipótesis de la habilidad como requisito para la sucesión. Recordemos, entonces, lo que dice Sarmiento sobre el tema. En su texto, aunque destaca que quien debía heredar el título de inca era el primogénito del anterior, construye en su redacción un considerable número de excepciones a la regla, básicamente, desde el gobierno de Huiracocha.
Por ello, en la versión de Sarmiento, ninguno de los que fueron designados por el inca logró convertirse en gobernante. Así, como destacó Pease (1991:97), en la versión de Sarmiento, Huiracocha designó a Inca Urco pero Pachacútec se hizo con el poder; este designó a Amaru Túpac Inca aunque Túpac Inca Yupanqui se convirtió en gobernante. Posteriormente, Túpac Yupanqui designó a Cápac Huari, pero fue Huaina Cápac quien se hizo con la borla y, finalmente, Huaina Cápac designó a Ninan Cuyochi, quien murió prematuramente dando lugar a las conocidas guerras entre Huáscar y Atahualpa (Sarmiento 1988 [1572]).

La pregunta sería, entonces, si realmente el cronista quería acomodar los datos para hacer parecer una sucesión dinástica o si, por el contrario, con estas informaciones sobre incas -elegidos por habilidad-, buscaba incorporar a la historia del Tahuantinsuyo la idea de una sucesión, bastante antigua, de herederos ilegítimos.

Un acercamiento alternativo al tema es el que nos ofreció Concepción Bravo (1976) cuando estudió la descendencia y la sucesión del Inca Huaina Cápac. En este trabajo, siguiendo la obra de Agustín de Zárate, la autora muestra cómo se había modificado la versión original del cronista, quien ofrecía un sistema distinto al hasta ahora señalado. En este texto primitivo de Zárate de 1555, señala la autora:

cuando un rey moría no era ninguno de sus hijos quien le sucedía inmediatamente, sino el mayor de sus hermanos. Después de la muerte de éste la sucesión retornaba al hijo mayor del rey precedente, y de éste a su hermano, después de este hermano al hijo mayor del anterior y así sucesivamente (Bravo 1976:108).

El tema planteado por Bravo, como se señaló, hace necesario profundizar en la información que ofrecen sobre la sucesión incaica las dos ediciones de Agustín de Zárate realizadas en el siglo XVI, cuyas incongruencias sobre el tema en cuestión -notadas anteriormente por Marcel Bataillon (1963), Concepción Bravo (1976), Pierre Duviols (1964), y Franklin Pease G.Y. en la edición que hace del mencionado cronista (Zárate 1995)- nos ofrecen un camino interesante. En este caso, como es conocido por todos, la célebre Historia del descubrimiento y conquista del Perú se editó por primera vez en Amberes, en 1555; y, con ciertos cambios, apareció 
en Sevilla una segunda edición en 1577. El asunto es más que evidente: en la segunda edición se cambiaron capítulos referentes a temas controversiales para la versión sobre la historia y las costumbres andinas, aparentemente con el propósito de perpetuar la imagen histórica que presentaban los llamados textos toledanos. De hecho, uno de los capítulos que se transformó por completo es el de la sucesión entre los incas. Analicemos las dos versiones del texto de Zárate:

Texto de 1555: Estos yngas començaron a poblar la ciudad del Cuzco, y desde allí fueron sojuzgando toda la tierra y la hicieron tributaria, sucediendo por línea derecha de hijos el imperio, como quiera que entre los naturales no suceden los hijos, sino primero el hermano del muerto siguiente en edad, y después de aquel fallecido torna el señorío al hijo mayor de su hermano, assídende en adelante hereda el hermano déste; y después torna a su hijo, sin que jamás falte este género de sucesión [énfasis mío] (1995 [1555]:XIII-XIV).

Texto modificado en 1577: Estos ingas comenzaron a poblar la ciudad del Cuzco, y desde allí fueron sojuzgando toda la tierra y la hicieron tributaria; y de ahí adelante iban sucediendo en este señorío el que más poder y fuerzas tenía, sin guardar orden ligítima de sucesión, sino por vía de tiranía y violencia; de manera que su derecho estaba en las armas [énfasis mío] (Zárate 1995 [1577]:XIV).

Claramente, como anotó anteriormente Pierre Duviols y resaltó Franklin Pease G.Y., el texto original de Agustín de Zárate fue modificado para dar la imagen de un sistema desordenado y sujeto a tiranía; coherente, además, con las distintas herramientas textuales que el virrey Toledo estaba preparando con miras a afianzar su visión sobre el Tahuantinsuyo. No es, por tanto, antojadizo suponer que la idea de la habilidad como único requisito para la transmisión del mando entre los incas sea una realidad más toledana que andina. En este caso, la pregunta por la sucesión quedaría nuevamente abierta en búsqueda de nuevas luces que nos permitan concordar lo que dice la historiografía sobre el sistema de sucesión de hermanos que planteaba la original versión de Zárate sobre el asunto. Sigamos, a partir de otros textos, las pistas de Zárate.

Siguiendo esta línea, aun cuando Hernando de Santillán afirme que sucedían los hijos a los padres y que, si había más de uno en la mujer principal, la designación priorizaba al más capaz entre los hijos del Inca, nuestro cronista desliza también información acerca del paso del mando al hermano del inca anterior:

[...] cuando el inga tenía muchos hijos, no venía la subcesion forzozamente al mayor, sino á aquel quel padre quería más y quería dejalle por rey $[\ldots]$ y siempre escogía el inga entre sus hijos el que era más hombre, ó el que habia en alguna hermana suya ó mujer de su linaje. Cuando sucedía morir el inga, sus hijos, los ingas orejones ó personas principales escogían otro hermano suyo y le daban la borla y lo alzaban por rey, como lo hicieron con Mango Inca por muerte de Guascar; y agora muerto Xayre Topa, los orejones questan tras la cordillera alzaron á su hermano Amaro Inga, al cual tienen allí por señor conforme a sus fueros y costumbres (Santillán 1968 [1563]:387).

La información que nos ofrece el texto de Hernando de Santillán acerca de la sucesión entre los incas es, como puede apreciarse, confusa. El autor combina en su alternativa sobre el tema una sucesión dinástica en la que el mando se transmite de padres a hijos, aunque no necesariamente a los mayores, pues, junto al criterio de habilidad como discriminador entre los candidatos, operaba el sistema que ofrecía Agustín de Zárate acerca de una sucesión entre hermanos como primera opción antes de pasar a la siguiente generación. Páginas más adelante, el mismo Santillán, al comentar la forma en que se elegía a los curacas, afirma que el tema era complicado y sugiere la elección de las autoridades locales por manos del inca. Sin embargo, reafirmando la complejidad del sistema señala que:

Acerca desto hay alguna variedad, porque otros dicen que á estos señores principales y curacas de pachaca y guaranga los sucedían sus hermanos el que dellos era más bastante para mandar, y esto aunque dejase hijos; y despues de muerto el hermano sucedían 
los hijos del primer hermano difunto y á éste sus hermanos, y por esta órden iba la sucesión, y al presente se hace desta manera; y esto parece ser así por muchas probanzas é informaciones que dello se han visto en la audiencia (Santillán 1968 [1563]:387-388).

Mencionamos anteriormente cómo en las llamadas Informaciones sobre los Incas, realizadas en Cuzco en la segunda mitad del siglo XVI, los funcionarios virreinales estaban decididos a presentar un sistema sucesorio caótico tanto para los gobernantes del Cuzco como para los curacas andinos. Así, las propias preguntas estaban destinadas a recoger respuestas que confirmaran su contenido. De este modo, los funcionarios españoles estaban interesados en mostrar que antes de los incas no hubo señores naturales, sino behetrías; y que los incas eran gobernantes ilegítimos que tiranizaron a los curacas, a quienes ponían en el gobierno sin una regla clara, a su voluntad. Sin embargo, hay un hecho evidente en el propio texto: todos los curacas entrevistados afirman ser hijos o nietos de los antiguos gobernantes; cuestión que, como es evidente, contradice incluso sus propias informaciones. Asimismo, cuando hablan del tema sucesorio, los propios curacas sugieren un sistema similar al que venimos presentando a partir de la información de Zárate. Vemos algunos ejemplos: "[...] diego chullo ycra e ques curaca del pueblo de lango de la encomienda de la menor del ynga y que por ser ya viejo a dexado el mando de su cacicazgo a un hermano suyo que tiene masfuerça [énfasis mío] quel para gouernar [...]" (Levillier 1940:105). Ese es, también, el caso de Domingo Xuxso natural de chibaco, quien menciona que "[...] su padre heramandon de diez yndios puesto por guainacapac en el dicho pueblo e que despues de muerto su padre por que este testigo no tenia abilidad fue puesto un hermano del dicho su padre [énfasis mío] para que los mandase [...]" (Levillier 1940:114). Asimismo, Don Francisco Suy Guaman, quien afirmaba ser curaca de cien indios, aseguraba que "[...] por ser viejo dejó de mandar y los manda el día de hoy un Don Alonso Pomaescoleo, su hermano [énfasis mío]" (Levillier 1940:134-135). Finalmente, un caso también similar es el de Don Francisco Vichic, natural de Hanan guanca, quien luego de asegurar que el padre fue también curaca -en este caso de quinientos indios- afirmaba que, muerto el padre, "[...]por faltarle la edad y memoria de los gobernar ha subcedido un hermano suyo [énfasis mío] en el cargo [...]" (Levillier 1940:159).

Habría que mencionar que, en todos los casos presentados, el tema de la habilidad como requisito para gobernar es señalado, exclusivamente, como una práctica asociada con los curacas y que, aparentemente, los testimonios presentados sugieren un sistema de reemplazo generacional. En ese caso, podría pensarse también para los incas en un sistema que combinaba dos posibilidades: en primer lugar, el reemplazo del gobernante por un hermano cuando se trataba de un momento en el que la siguiente generación aún no estaba "preparada"; y, en segundo lugar, en una transmisión del mando a la siguiente generación cuando esta ya había llegado a lo que podría considerarse como una suerte de "edad propicia".

De hecho, como veremos a continuación, parecería que el tema sucesorio combinaba múltiples posibilidades según el contexto en el que ocurría la necesidad de reemplazar al gobernante. Sobre el tema, el Inca Garcilaso de la Vega también señala esta multiplicidad de posibilidades afirmando que, aun cuando la primogenitura era la regla que se debía aplicar en primer lugar, y que, de no existir hijos en la coya, podría heredar la borla un hijo del inca en una mujer secundaria. Da cuenta, también, de la posibilidad de transmitir el mando al "más capaz", e incluso a un hermano del gobernante:

En otras heredaba el hijo más bienquisto de sus vasallos, amado por su virtud y afabilidad, que parece elección más que no herencia $[\ldots]$ En otras provincias heredaban todos los hijos por su antigüedad, que muerto el padre, sucedía el hijo mayor, y luego el segundo y tercero, etc. [...] De haber oido esta manera de heredar de algunos curacas se engañó un historiador español, diciendo que era común costumbre en todo el Perú, no solamente en los caciques, mas tambien en los reyes heredar los hermanos del rey, y luego los hijos de ellos por su orden y antigüedad: lo cual no hubo en los reyes Incas sino en algunos curacas como hemos dicho (Garcilaso 1965 [1609]:129-130).

Vistas las referencias mencionadas hasta el momento, vale la pena recordar que, pese a resaltar la habilidad en el mando, desde sus planteamientos 
iniciales (1953), María Rostworowski notó la existencia en los textos coloniales del sistema alternativo que venimos comentando -tema que reelaboró en 1961 destacando que la sucesión del hermano, antes que del hijo, fue una práctica asociada con las etnias de la costa norte, aunque posteriormente ampliada para otras poblaciones serranas y costeñas, mas nunca aplicada para los incas (Rostworowski 1953, 1961, 1983:155)-. La práctica de heredar entre hermanos se dio también para el caso del Tahuantinsuyo, por lo menos, claramente entre los incas posteriores a Huaina Cápac; sobre todo, entre los llamados incas de Vilcabamba, entre los que, incluso, encontramos casos de hijos del inca en las llamadas "mujeres secundarias", como el caso de Paullu Inca. Así las cosas, se puede afirmar que, tanto en el caso de los curacas como en el de los incas, era claro que ambos gobernantes -el de Hanan y el de Rurin- debían pertenecer a la misma generación, y que su reemplazo se daba en forma simultánea. En ese sentido, Concepción Bravo señala claramente cómo los tres gobernantes Hanan anteriores a Huaina Cápac dejaron el gobierno y fueron sustituidos por sus hijos dentro de un proceso en el que el acceso al poder se hace luego de un periodo de aprendizaje, como correinantes. De ese modo, para el caso de los incas, estaríamos ante un sistema sucesorio múltiple, que no dejaba dudas sobre la situación y que preveía cualquier contexto (Bravo 1992:17).

El sistema incluía el reemplazo de los gobernantes Hanan y Rurin cuando la edad de los gobernantes había llegado a un punto en el que era mejor que abandonaran el ejercicio cotidiano del poder. Por esto, existía una suerte de periodo de prueba en el que los aspirantes hacían valer sus derechos demostrando su habilidad para gobernar, prefiriéndose a los hijos del inca en la coya que a los otros. Ello se realizaba tomando en cuenta que eran considerados como hijos del inca todos los integrantes de la más alta nobleza incaica pertenecientes a una determinada generación. Como es evidente, y como nos hace pensar la información colonial, ante la ausencia de herederos de esta, podía hacerse con la borla alguien de rango inferior (un hijo del inca en una mujer de la nobleza provincial, por ejemplo), como en el caso de Paullu Inca. Evidentemente, en la consolidación de un candidato como inca intervenían -además de los criterios de "derecho" mencionados- aquellos que correspondían a la llamada "habilidad" para gobernar y en la que intervenían tanto la aprobación del grupo mostrada en la celebración de múltiples rituales, como las convenientes alianzas que estos pudieron hacer con los linajes de su madre y esposa.

Una descripción de este sistema complejo -aunque para nada caótico, como quiso demostrar la posición toledana- es la que se dio en 1563 en Lima, según registran los documentos publicados por Roberto Levillier. En este caso, se da cuenta de la sucesión de los curacas de Hanan Guanca. Esta misma describe la complejidad que venimos anunciando y que, aun cuando se trata de un caso colonial y "provincial", nos puede dar mayores detalles sobre el tema, incluso para los incas. En este caso, se menciona con el permiso de las autoridades coloniales:

[...] e otro si dixo su Excelencia que porque le pareciaquel dicho don Carlos al presente no tenya hedad complida para poder gouernar los dichos yndiosmandava que en el entretanto que la tiana use el dicho don hernando su tio que presente estava al qual manda que lo hizieseasy y a los dichos yndios y principales que le obedesciesen e acatasen en el dicho entretanto bien asy como avian de obedecer y acatar al dicho don Carlos sy fuera de hedadcomplida y quel dicho don hernando en el dicho ynterin gozase de todas las preheminenciasquel dicho don Carlos avia de gozar y asimismo su Excelencia mando que dicho don hernando se sentase en otro duho junto con el dicho don Carlos el qual se sento e luego se levantaron ambos y fueron a besar las manos de su Excelencia y Su Excelencia dio al dicho Carlos y don hernando de su mano dos pares de ropas de las que dichos yndios suelen traer para que las tuviesen por insignias del dicho cargo e ymbistidura [...] (Leviliier 1940:96-98).

El texto sugiere así una herencia del cargo de hermano a hermano como un mecanismo previo al paso del poder a la siguiente generación. Evidentemente, habría que entender el término "hermano" en su consideración andina; esto es, como contemporáneos. Bajo este esquema, podría perfectamente entenderse el complejo proceso sucesorio que presentaba Betanzos, y que comentábamos líneas atrás, al mencionar la transmisión del mando entre Túpac Inca, Yamque Yupanqui y Huaina Cápac. 
Finalmente, sobre el tema del correinado y la probable elección de dos incas, uno para Hanan Cuzco y otro para Rurin Cuzco, bien podría entenderse que, de los dos candidatos que aparentemente siempre quedaban en el proceso sucesorio, uno de ellos -el que finalmente se identificaría como jefe de los Rurin Cuzco- era identificado como "segundo hijo" del inca, con lo cual quedaba la condición de "primer hijo" para el Inca Hanan. De esta forma, la aparente confusión que generó para los españoles el entendimiento de este complejo sistema fue utilizada para elaborar una conveniente imagen de caos sucesorio de modo que se lograba un argumento adicional en el proceso de justificación de la conquista.

Agradecimentos: Agradezco a quienes revisaron el artículo por encargo de la Revista, ya dado que con sus comentarios fue posible enriquecer mi manuscrito.

\section{Referencias Citadas}

Anónimo de Yucay 1979 [1570]. Dominio de los Yngas en el Perú y del que Su Magestad tiene en dichos Reynos. En Historia y Cultura, editado por J. Chinese, vol. 4, pp. 105-152. Museo Nacional de Arqueología, Antropología e Historia del Perú, Lima.

Betanzos, J.1987 [1557]. Suma y Narración de los Incas. Versión y estudio preliminar de María del Carmen Martín Rubio. Ediciones Atlas, Madrid.

Bravo, C.1976. Los Matrimonios de Huayna Cápac: su sucesión, según diversas fuentes. Actas del XLI Congreso Internacional de Americanistas 2:107-116. México.

- - - - 1992. Del poder dual a la diarquía en el Estado Inca. Revista Complutense de Historia de América 18:11-61.

Cerrón-Palomino, R. 2002. Hurin: un espejismo léxico opuesto a Hanan. Actas del IV Congreso internacional de Etnohistoria 1.Fondo Editorial Pontificia Universidad Católica del Perú, Lima.

D’Altroy, T. 2003. Los Incas. Ariel, Barcelona.

Duviols, P. 1964. La historia del descubrimiento y de la conquista del Perú de Agustín de Zárate. Remaniée Conforménment aux vues Historico-politiques du Vice-Roi Toledo. Annales de la Faculté des Letresd'Aix36:151-155.

Garcilaso de la Vega, I. 1960 [1609]. Comentarios Reales de los Incas. En Obras Completas. Edición y estudio preliminar de Carmelo Sáenz de Santa María S.J. Biblioteca de Autores Españoles, Madrid.

Guaman Poma de Ayala, F. 1993 [1613]. Nueva Corónica y Buen Gobierno. Edición y prólogo de F. Pease G.Y., Vocabulario y traducciones de J. Szemiñski. Fondo de Cultura Económica, Lima.

Hernández Astete, F. 1997. Roles Femeninos en la Organización de la Élite Incaica. Tesis para optar el título de Licenciado en Historia, Facultad de Letras y Ciencias Humanas, Pontificia Universidad Católica del Perú, Lima.

- - - 1998. Roles sexuales en la organización incaica. Histórica 22:93-134. Pontificia Universidad Católica del Perú, Lima.

- - - - 2002a. La Mujer en el Tahuantinsuyo. Pontificia Universidad Católica del Perú, Lima.

- - - 2002b. El poder incaico: una aproximación a la Figura del Inca hurin. En El Hombre y los Andes: Homenaje a Franklin Pease G.Y., editado por R. Varón y J. Flores vol. 2, pp. 589-596. Pontificia Universidad Católica del Perú, Lima.

- - - 2010. La Élite Incaica y la Articulación del Tahuantinsuyo. Biblioteca Digital Complutense. Universidad Complutense de Madrid, Madrid.

Levillier, R.1940. Don Francisco de Toledo. Supremo Organizador del Perú. Su Vida, su Obra (1515-1582). Sus Informaciones sobre los Incas (1570-1572). Tomo II. Buenos Aires.
Pachacuti Yamqui Salcamaygua, J.1993. Relación de Antigüedades deste Reyno del Pirú. Editado por Pierre Duviols y César Itier. Instituto Francés de Estudios Andinos-Centro de Estudios Regionales Andinos Bartolomé de Las Casas, Lima-Cusco.

Pizarro, P. 1986. Relación del Descubrimiento y Conquista de los Reinos del Perú. Pontificia Universidad Católica del Perú, Lima.

Pease, F. 1991. Los Últimos Incas del Cuzco. Alianza Editorial, Madrid.

Regalado de Hurtado, L. 1996 [1993]. Sucesión Incaica. Aproximación al Mando y Poder entre los Incas a partir de la Crónica de Betanzos. Pontificia Universidad Católica del Perú, Lima.

Rostworowski, M. 1961. Curacas y Sucesiones. Costa Norte. Lima. - - - - 1983. Estructuras Andinas del Poder. Instituto de Estudios Peruanos, Lima.

- - - 1988. Historia del Tawantinsuyu. Instituto de Estudios Peruanos, Lima.

- - - 2001 [1953]. Pachacútec. Obras Completas. Instituto de Estudios Peruanos, Lima.

Rowe, J. 1963 [1946]. Inca Culture at the time of the Spanish Conquest. En Handbook of South American Indians, II. Cooper Square Publishers, Washington DC., New York.

Santillán, H. 1968 [1563]. Relación del Origen, descendencia, Política y Gobierno de los Incas. Primera serie. Tomo III. Editores Técnicos Asociados S.A., Biblioteca Peruana, Lima.

Sarmiento de Gamboa, P. 1988 [1572]. Historia de los Incas. Biblioteca de viajeros hispánicos, Miraguano Ediciones, Ediciones Polifemo, Madrid.

Someda, H. 1999. El Imperio de los Incas. Imagen del Tahuantinsuyu creada por los Cronistas. Pontificia Universidad Católica del Perú, Lima.

Zárate, A. 1995 [1577]. Historia del Descubrimiento y Conquista del Perú. Pontificia Universidad Católica del Perú, Lima.

Ziólkowski, M. 1997. La Guerra de los Wawqi: los Objetivos y los Mecanismos de la Rivalidad dentro de la Élite Inka, S. XV$X V I$. Ediciones ABYA-YALA, Quito.

Zuidema, R. 1980. El sistema de parentesco incaico: una nueva visión teórica. En Parentesco y Matrimonio en los Andes, editado por E. Mayer y R. Bolton. Pontificia Universidad Católica del Perú, Lima.

- - - 1995 [1964]. El Sistema de Ceques del Cuzco. Pontificia Universidad Católica del Perú, Lima. 


\section{Notas}

1 La oposición Hanan Cuzco-Rurin Cuzco estaba asociada con las dos mitades en las que se dividía la nobleza cuzqueña. Elegimos la voz Rurin y no Hurin siguiendo a Cerrón-Palomino (2002), quien afirma que este término (hurin) nunca existió y sería una suerte de espejismo léxico desarrollado en oposición a 'Hanan'.

2 Una aplicación de la idea de la habilidad como principal mecanismo para la sucesión incaica puede encontrarse en los trabajos de: D'Altroy (2003), Someda (1999), Ziólkowski (1997) y Zuidema (1995 [1964]), entre otros.

3 La tradición de "rogar" a la momia para acceder al matrimonio con alguna de las mujeres de su aillu o panaca es presentada, también, por Pedro Pizarro cuando indica que tuvo que intervenir en el ritual para interceder por Manco Inca ante una orden de Francisco Pizarro: “[...] me lleuaron a un bulto de estos muertos, donde estaua asentado dentro de unas andas, que así lo tenían; y el yndio diputado que hablaua por él de un lado, y la yndia al otro, sentados cabe el muerto" y Pizarro rogó, el indio miró a la india y finalmente aceptaron" (Pizarro 1986 [1571]:53-54).

4 En otra ocasión, se ha demostrado cómo la celebración de matrimonios del inca, tanto con mujeres de la élite cuzqueña como con las hijas y hermanas de los curacas, fue uno de los mecanismos que sustentaron el poder del gobernante del Cuzco (Hernández Astete 1997, 2002a).
5 Para un análisis detallado del caso, puede consultarse Regalado de Hurtado(1996 [1993]:39) y Hernández Astete (1998:111). Es importante mencionar que la mujer en cuestión es nada menos que Angelina Yupanqui, esposa de Pizarro y posteriormente del propio Betanzos.

6 Alejandro Ortiz, Comunicación personal en el año 2003.

7 Guaman Poma de Ayala, en su crónica, luego de redactar las biografías de Incas y Coyas, hace lo propio con los que llama "capitanes". Al parecer, los hijos de los anteriores. En un trabajo anterior (Hernández Astete 2010), se ha propuesto que esta institución funcionaba como una suerte de entrenamiento para los probables gobernantes.

8 Regalado de Hurtado1996 [1993) afirma que una tarea asociada al inca luego del proceso sucesorio es precisamente la edificación.

9 Se menciona que, posteriormente, Amaru Túpac derrotó a los asesinos de su hermano. Sin embargo, luego de la primera derrota, no aparece más como aspirante a inca.

10 Para mayor detalle sobre la doble dualidad de incas y coyas en la obra de Guaman Poma, revisar Hernández Astete (1998:123, 2002b)

11 Este hecho es narrado también por Betanzos, aunque para él, tras la negativa de Pachacútec de alzarse con el poder, los señores del Cuzco presionan a Huiracocha Inca y es este quien convierte en inca al vencedor de los Chancas, dándole el nombre de PachacutiYngaYupangueCapacIndichuri. 
